\section{Virulência in vitro por leveduras isoladas da região cérvico-vaginal de cadelas}

\section{In vitro virulence of yeasts isolated from the cervicovaginal region of bitches}

\author{
Denise dos Santos Navarro Antonio', Daniel Paiva Barros de Abreu², Mario Mendes Bonci³ , Mario Tatsuo Makita ${ }^{3}$, \\ Sergio Gaspar de Campos ${ }^{4}$ \& Francisco de Assis Baroni ${ }^{4 *}$ \\ 'Médico veterinário, Mestre em Medicina Veterinária. Programa de Pós-graduação em Medicina Veterinária, Universidade \\ Federal Rural do Rio de Janeiro - UFRRJ, Seropédica, RJ, Brasil \\ ${ }^{2}$ MSc. Programa de Pós-graduação em Ciências Veterinárias - PPGCV, Universidade Federal Rural do Rio de Janeiro - UFRRJ, \\ Seropédica, RJ, Brasil \\ ${ }^{3}$ Médicos veterinários. Programa de Pós-graduação em Medicina Veterinária - PPGMV, Universidade Federal Rural do Rio de \\ Janeiro - UFRRJ, Seropédica, RJ, Brasil \\ ${ }^{4}$ Doutores. Departamento de Microbiologia e Imunologia Veterinária, Instituto de Veterinária, Universidade Federal Rural do \\ Rio de Janeiro - UFRRJ, Seropédica, RJ, Brasil
}

\section{Resumo}

Objetivou-se verificar as espécies de leveduras da mucosa cérvico-vaginal de cadelas e a virulência "in vitro" dos isolados por ensaios de produção de protease e fosfolipase. Utilizou-se 40 fêmeas caninas, divididas em não ovariectomizadas e cadelas gestantes. As coletas ocorreram por lavado cérvico-vaginal, com tampão salina estéril, seguindo-se isolamento em meio Ágar Infuso Cérebro e Coração, sucedido de provas bioquímicas para identificação das leveduras. A avaliação da produção de protease e de fosfolipase foi realizada "in vitro", com meios específicos. Das 40 cadelas, em 82,5\% obteve-se o crescimento fúngico. Foram obtidos 60 isolados, sendo 21 (35,0\%) identificados como Candida spp, 19 (31,66\%) Malassezia spp, 13 (21,66\%) Rhodotorula spp e 7 (11,66\%) Trichosporon spp. Das 60 cepas testadas para produção de protease $36,66 \%$ foram negativas, 33,33\% positivas e 30,0\% forte positivas. Para produção de fosfolipase, $45,0 \%$ foram negativas, $38,33 \%$ forte positivas e $16,66 \%$ positivas. A maioria dos isolados apresentou algum grau de virulência se considerarmos a capacidade de produção destas enzimas. O isolamento de cepas que são possíveis agentes de distúrbios reprodutivos, a determinação das espécies e a avaliação dos fatores de virulência representam talvez a solução de inúmeros distúrbios ainda pouco explicados e controlados na medicina veterinária.

Palavras-chave: produção enzimática, fungos, micobiota, trato reprodutivo.

\begin{abstract}
The present study verified the species of yeast present in cervicovaginal mucosa of bitches and the in vitro production of virulence factors. A total of 40 female dogs were divided into not neutered and pregnant groups and samples were collected implementing cervical-vaginal lavage with sterile saline. Isolation was performed in Brain and Heart Infusion Agar, followed by morphological, biochemical and physiological evaluations for phenotypical identification of yeasts, and determination of phospholipase and protease production using specific mediums. Fungal growth was obtained in 33 animals (82.5\%), totalizing 60 isolates. Candida spp was the most prevalent genus, with 21 isolates (35.0\%), followed by 19 (31.66\%) Malassezia spp, 13 (21.66\%) Rhodotorula spp, and 7 (11.66\%) Trichosporon spp. For the evaluation of protease production, $36.66 \%$ were negative, while $33.33 \%$ positive and $30.0 \%$ strong positive. For phospholipase production $45.0 \%$ were negative, $16.66 \%$ positive, and $28.33 \%$ strong positive. Most of the isolates showed some degree of virulence, considering the production of these two enzymes, and were previously described as infectious agents of the reproductive tract. A better understanding of the genital tract microbiota could potentially clarify some reproductive disorders in small animal practice.
\end{abstract}

Keywords: enzymatic production, fungi, reproductive tract.

\section{Introdução}

O crescimento significativo da população canina nas últimas décadas levou a um aumento e diversificação da atuação do Médico Veterinário, principalmente pelo aumento da popularidade de cães de raça, bem como pela valorização afetiva e comercial dos animais de companhia. \section{BJ M

Como citar: Antonio, D. S. N., Abreu, D. P. B., Bonci, M. M., Makita, M. T., Campos, S. G., \& Baroni F. A. (2017). Virulência in vitro por leveduras isoladas da região cérvico-vaginal de cadelas. Brazilian Journal of Veterinary Medicine, 39(1), 61-67. doi: 10.29374/2527-2179.bjvm0888

Fonte de financiamento: $O$ projeto foi parcialmente financiado pela CAPES concessão de bolsa de mestrado.

Conflito de interesses: Os autores declaram não haver conflito de interesses que precisam ser informados.

\section{Recebido: Janeiro 25, 2017}

Aceito: Março 12, 2017.

O estudo foi realizado na Universidade Federal Rural do Rio de Janeiro - UFRRJ, Seropédica, RJ, Brasil.

\section{*Correspondência}

Francisco de Assis Baroni

Departamento de Microbiologia e Imunologia Veterinária, Instituto de Veterinária,

Universidade Federal Rural do Rio de Janeiro - UFRRJ

BR 465, Km 07, Zona Rural CEP 23890-000 - Seropédica (RJ), Brasil E-mail: baroni@ufrrj.br 
A reprodução destes animais é uma área da Medicina Veterinária que muito vem se desenvolvendo, embora diversos aspectos reprodutivos necessitem ser esclarecidos para que as biotécnicas possam ser aplicadas com sucesso, ampliando o ganho reprodutivo, principalmente de cães e gatos. Diferentes problemas são atualmente observados na reprodução canina, destacando-se aqueles que levam à infertilidade verdadeira, anestro persistente ou baixa prolificidade, relacionados a distúrbios hormonais, afecções uterinas e ovarianas, defeitos congênitos ou adquiridos do aparelho reprodutivo e doenças infecciosas.

A microbiota cérvico-vaginal pode ser considerada de residente para transitória, ligada diretamente ao ambiente e manejo. Em condições normais possui composição e número variável, podendo apresentar crescimento exacerbado em animais com sistema imunitário comprometido, culminando em infecções clínicas e subclínicas. No campo da microbiologia do trato reprodutivo feminino, conhecer os micro-organismos saprófitas e diferenciar agentes patogênicos de constituintes normais da microbiota pode ser considerado um requisito básico, uma vez que podem tornar-se responsáveis por infecções. O isolamento de micro-organismos da microbiota endógena, considerada pouca virulenta, não pode ser desprezado, pois é possível que seus constituintes sejam agentes etiológicos de afecções ou tenham importância clínica.

Dentre os fungos, leveduras pertencentes aos gêneros Candida, Malassezia e Rhodotorula entre outras, foram descritas como pertencentes à microbiota de mucosas e pele de mamíferos (Rippon, 1988), podendo tornar-se patogênicas, pela exacerbação de seu crescimento ou mecanismos de defesa comprometidos.

O conhecimento da microbiota cervico vaginal de cadelas é pequeno principalmente quando consideramos a presença de fungos filamentosos e leveduras, desconhecendo-se o poder de virulência destas leveduras. Tais fatos estimularam o desenvolvimento deste trabalho.

\section{Material e métodos}

A coleta de material da mucosa cérvico-vaginal foi realizada em animais de locais distintos como o Projeto Castração e Projeto S.O.S. (da UFRRJ) e propriedades particulares do município do Seropédica. O isolamento, identificação, caracterização fenotípica e testes de virulência foram realizados no Laboratório de Leveduras Patogênicas e Ambientais (LLPA) - Departamento de Microbiologia e Imunologia Veterinária no Instituto de Veterinária da UFRRJ - (DMIV-IV-UFRRJ). Foram utilizadas 40 fêmeas caninas a partir de seis meses de idade, sem distinção de raça, sendo um grupo constituído de cadelas não castradas $(n=20)$ e outro de cadelas gestantes $(n=20)$. Os animais foram submetidos a exames clínicos e complementares (hemograma com pesquisa de hemoparasitos e bioquímica renal e hepática) para verificar a higidez. O material foi obtido por meio um lavado cérvico-vaginal, com tampão salina estéril (Fraga et al., 2008), sendo os lavados transportados sob refrigeração ao laboratório. Cada lavado foi semeado na superfície de meio de cultura Ágar Infuso de Cérebro e Coração (BHIA), com 0,01\% de cloranfenicol, contido em placas de Petri. Utilizou-se inóculos de $1 \mathrm{~mL}$ de cada amostra pela técnica "pour plate", em triplicata, com incubação a $25^{\circ} \mathrm{C}, 32^{\circ} \mathrm{C}$ e $37^{\circ} \mathrm{C}$. As leituras foram realizadas do terceiro ao quinto dia de inoculação.

Aidentificação foi realizada prioritariamente segundo Kurtzman \& Fell (1998) e Kurtzman, Fell \& Boekhout (2011). Foi utilizado o protocolo de identificação de leveduras do LLPA, verificando-se aspectos macro e micromorfológicos, seguindo-se estudos das características fisiológicas e bioquímicas. Em alguns casos, utilizamos Chromogar ${ }^{\circledR}$ e o Sistema Candfast ${ }^{\circledR}$, como auxiliares.

Nas placas de Petri em que o lavado cérvico vaginal foi inoculado $(1,0 \mathrm{ml})$ foi realizada a contagem total de leveduras com auxílio da lupa. As Unidades Formadoras de Colônias (UFC) foram contabilizadas, realizando-se o cálculo por mL da amostra. Nos casos em que houve crescimento exacerbado da levedura (> 300 colônias/ placa), procedeu-se à feitura de diluições seriadas, efetuando-se nova contagem de colônias e multiplicação pelo fator de diluição (Markey et al., 2013).

A pesquisa de produção de protease foi realizada em meio protease, contendo soroalbumina bovina (Fração V, Sigma) e o teste de produção de fosfolipase, foi realizado em meio Agar fosfolipase (Price et al., 1982) que apresenta em sua composição, gema de ovo e $\mathrm{CaCl}_{2}$. Os meios preparados foram vertidos para placas de Petri. Em ambos testes, as amostras foram ativadas por cultivo de 48 horas em meio Sabouraud, procedendo-se a semeadura na parte central da superfície do meio, sempre em duplicata, com incubação a $32^{\circ} \mathrm{C}$ e leituras do $3^{\circ}$ ao $15^{\circ}$ dia. As produções 
enzimáticas foram verificadas pela presença de um halo translúcido ou por uma zona de precipitação, respectivos à presença de protease ou de fosfolipase, respectivamente, ocorrente em torno do ponto de crescimento da colônia. A atividade proteolítica enzimática, denominada "Pz" de cada cepa testada, foi estimada pelo cálculo da razão entre o diâmetro da colônia (dc) e o diâmetro total formado pela colônia e pela zona de degradação (dcp), ou seja $\mathrm{Pz}=\mathrm{dc} / \mathrm{dcp}$. Uma régua transparente foi usada para mensurar os diâmetros das colônias e dos halos (Figura1). Os resultados considerados como definitivos foram classificados conforme Tabela 1. Os dados obtidos foram colocados em planilha Excel e analisadas pelo $\mathrm{X}^{2}$ (quiquadrado) e para permitir a análise estatística utilizou-se o programa Graph Test, 2005.

\section{Resultados e discussão}

Ocorreu crescimento fúngico no material ginecológico processado de 33 das 40 cadelas utilizadas (82,5\%). Foram obtidos um total de 60 isolados fúngicos, sendo 21 (35,0\%) identificados como Candida spp, 19 (31,66\%) como Malassezia spp, 13 (21,66\%) como Rhodotorula spp e 7 (11,66\%) como Trichosporon spp. Dos 19 isolados obtidos do gênero Malassezia, todos foram identificados como M. pachydermatis que foi a levedura mais representativa em número de espécies.

Cleff et al. (2007) isolaram leveduras da secreção vaginal de cadelas e obtiveram Candida spp em um número maior de isolados, seguido de Malassezia pachydermatis e de Rhodotorula spp, podendo-se então considerar os resultados concordantes com estes autores. Stein et al. (2009)

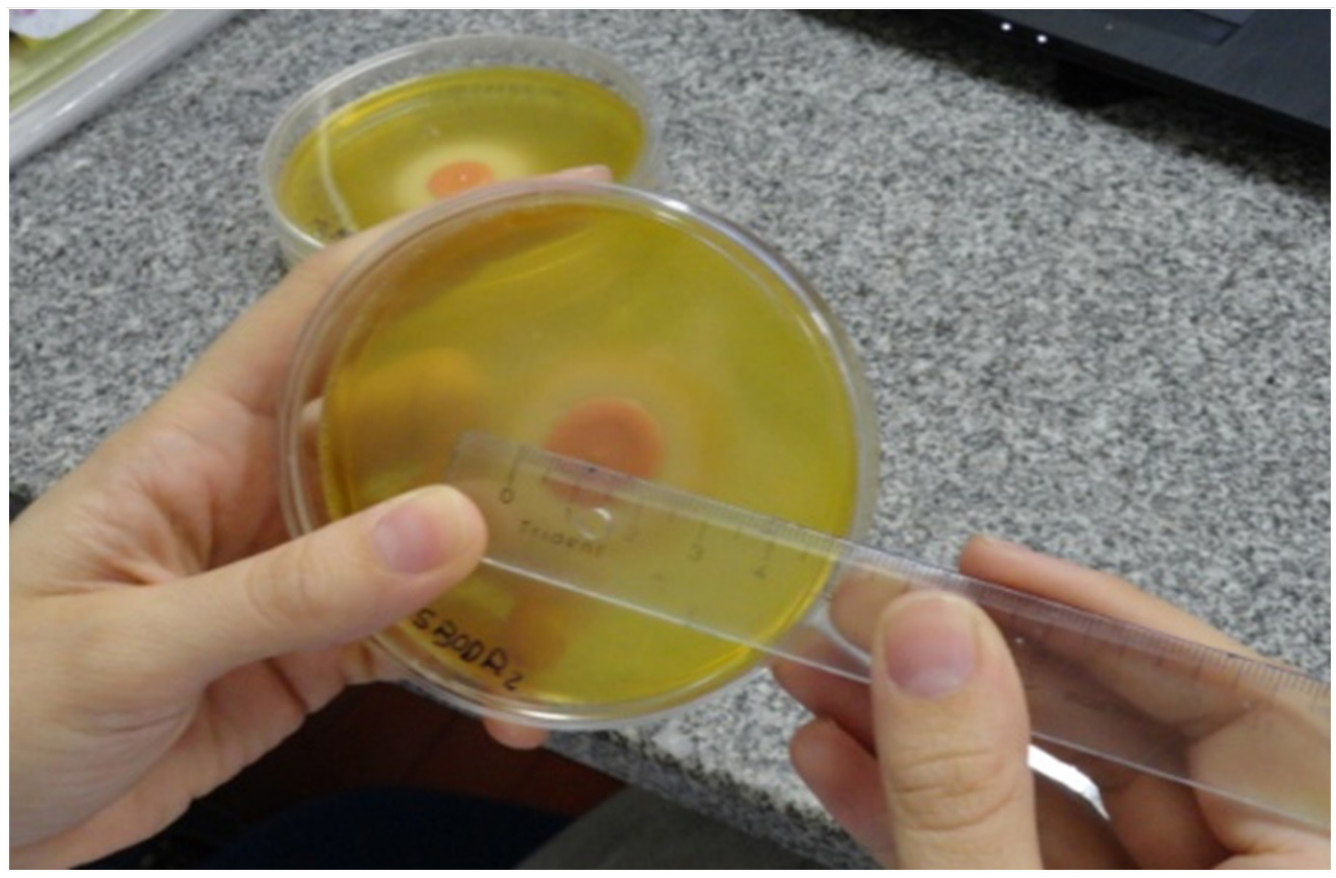

Figura 1. Meio de cultura para detecção da enzima fosfolipase mostrando colônia da levedura Rhodotorula spp e procedimento de medida da colônia e do halo de precipitação ao redor da mesma.

Tabela 1. Atividade de Protease e de Fosfolipase conforme o Pz e o código de acordo com Price et al. (1982).

\begin{tabular}{ccc}
\hline $\mathbf{P z}$ & Atividade Enzimática & Código \\
\hline$=1,0$ & Negativa & 1 \\
$>0,64<1,0$ & Positiva & 2 \\
$<0,64$ & Fortemente Positiva & 3 \\
\hline
\end{tabular}

Pz = relação entre diâmetro da colônia e diâmetro após formação de halo de precipitação ou halo de lise proteica. Códigos 1,2e 3 atribuídos respectivamente a produção enzimática negativa, positiva ou fortemente positiva. 
em trabalho similar, no entanto, observaram que das amostras positivas, Malassezia pachydermatis foi a levedura mais isolada, seguida de Candida spp, Rhodotorula spp associada com Candida spp, Trichosporon spp e Malassezia pachydermatis associada com Candida spp, havendo portanto uma discordância parcial. Também Fraga et al. (2008) relataram a presença de leveduras na mucosa vaginal sendo que as freqüências encontradas por eles diferem dos achados do presente estudo.

A presença de Candida spp, principalmente C. albicans, era previsível. A presença em grande número, no entanto é aspecto a ser sempre discutido, pois podem ocorrer quadros de candidíasee de candídide. A candidíase é um processo decorrente do crescimento exacerbado desta levedura devido a fatores como imunossupressão, tratamentos corticoterápicos, antibioticoterapia prolongada etc. Diversamente, a candídide é uma reação do tipo erupção dérmica secundária à presença de Candida spp, ocorrente sempre distante do local ou locais onde a levedura encontra-se presente, por hipersensibilidade a toxinas produzidas pela mesma. Existem hoje, na dermatologia veterinária, várias situações em que não se chega a um diagnóstico definitivo e que os animais sempre apresentam uma aparente recidiva no quadro clínico. Como estas lesões são estéreis, livres do micro-organismo, pode-se pensar nelas como uma possibilidade.

Malassezia pachydermatis é a espécie mais estudada em animais, eé considerada como parte da microbiota de vários sítios anatômicos de cães e gatos, sendo inclusive já relatada por alguns autores como parte da microbiota vaginal (Cleff et al., 2007; Stein et al., 2009). Embora M. pachydermatis apresente grande implicação em problemas dermatológicos em cães e inclusive quadros clínicos de otites, são poucos os relatos de seu crescimento no trato reprodutivo feminino destes animais.

Leveduras como Rhodotorula spp e Trichosporon spp apesar de menor incidência na região estudada, não devem ser desconsideradas uma vez que podem ser possíveis patógenos sob diferentes condições, desde o momento que o animal apresente uma susceptibilidade relativa ao sistema imune. É sabido que toda a microbiota normal do trato reprodutivo pode eventualmente tornar-se patogênica e Rhodotorula é apenas um destes agentes, tendo sido relacionada a casos de abortamentos e de infertilidade, assim como também já foram implicados Candida e Trichosporon (Verma et al., 1999). Portanto, devido aos relatos destas leveduras como causadoras de distúrbios em animais, o conhecimento de sua presença na região estudada fornece subsídios para compreendermos melhor situações de alterações numéricas ou de composição nas mucosas do trato reprodutivo.

No grupo 1, obteve-se o isolamento fúngico em 16 (80,0\%) das 20 cadelas, totalizando 22 isolados, enquanto que no grupo 2 , o isolamento foi em 17 (85,0\%) cadelas, totalizando 38 isolados. Em ambos grupos, as leveduras isoladas são as mesmas, diferindo na frequência. Malassezia spp foi a mais frequente no grupo 1, seguida de Candida spp, Rhodotorula spp e Trichosporon spp. No grupo 2 , Candida spp foi a amis frequente, seguida de Rhodotorula spp, Malassezia spp e Trichosporon spp. As leveduras isoladas são, portanto, as mesmas nos dois grupos, diferindo na frequência. Um maior aprofundamento poderia evidenciar se existem alterações, durante o ciclo estral que influenciem estas leveduras. Até o momento, poucos avaliaram a microbiota cérvico-vaginal de cadelas gestantes. Cleff et al. (2007) correlacionaram amostras de secreção vaginal de 14 cadelas onde 2 eram gestantes. Das 224 amostras, 71,4\% foram positivas para presença de Candida em cadelas gestantes. Esta amostragem, no entanto, ainda pode ser considerada pequena. Em nosso estudo, no entanto, a frequência maior de Candida spp no grupo 2 pode ser justificada por ser uma levedura oportunista, estando associada com a imunossupressão que ocorre no trato reprodutivo feminino no momento da implantação do feto e durante toda a gestação. Ocorrem várias modificações no trato genital inferior feminino durante a gestação, devido a estímulos hormonais tais como: hipertrofia das paredes vaginais, aumento do fluxo sanguineo e da temperatura, bem como uma maior exposição do epitélio colunar do colo por causa da hiperplasia glandular, com formação da ectopia característica da gestação. Essas alterações fisiológicas, apesar de terem função protetora ao útero, à gravidez e ao organismo materno, podem tornar a gestante mais suscetível às infecções vaginais (Tedesco et al., 2006).

Pode-se considerar, ainda, a possibilidade de inibição de alguns micro-organismos por toxinas "killer". Algumas leveduras produzem estas toxinas que tem capacidade de atuarem nas membranas celulares de outras, diminuindo a integridade por formação de canais permeáveis, ocasionando a morte celular. Algumas espécies de Pichia spp apresentam efeito deletério sobre 
Malassezia pachydermatis. Neste caso, o não isolamento de Pichia spp em nosso trabalho poderia justificar o maior isolamento de Malassezia pachydermatis.

Na contagem de células viáveis, das 38 amostras detectou-se média de aproximadamente $474,05 \pm 1$ leveduras/ ml da amostra. As médias das Unidades Formadoras de colônias entre os grupos apresentaram uma diferença significativa. Para o primeiro grupo a média foi de aproximadamente $24,1+1 / \mathrm{mL}$ e para o segundo de $981,22 \pm 1 / \mathrm{mL}$. Em duas amostras, oriundas de cadelas gestantes, devido ao número de colônias, houve necessidade de realizar a diluição seriada para a contagem das UFC.

O maior número de UFC do grupo 2 em relação ao grupo 1, provavelmente deve-se ao fato de, na gestação, ocorrerem varias alterações fisiológicas que afetam todo o organismo modificando seu metabolismo. A placenta é fonte de muitos fatores imunossupressivos; além dos hormônios estradiol e progesterona, algumas proteínas do soro fetal são imunorreguladoras, como isoformas da fetoproteína- $\alpha$, e também da placenta, como macroglobulina- $\alpha$ e interferon placentário. O líquido amniótico, por sua vez, é rico em fosfolipídeos imunossupressivos (Tizard, 1998).

Das 60 cepas testadas para produção de protease, 22 (36,66\%) foram negativas, 20 (33,33\%) foram classificadas como positivas e 18 (30,0\%) como forte positivas, ou seja, 38 (63,33\%) das amostras são produtoras de protease, com índices de Pz variando de 0,52 a 0,92 (Figura 1). Em relação aos grupos, não houve diferença significativa para produção. Treze cepas do grupo 1 (59,09\%) produziram protease e 24 (63,15\%) do grupo 2 igualmente foram produtoras.

As proteases são consideradas importante fator de virulência por auxiliarem o fungo na invasão tecidual e disseminação dentro do hospedeiro (Kumar et al., 2006). Diversos estudos têm relatado a forte correlação entre a secreção de protease e o grau de virulência das cepas (Corrêa et al., 2009; Bona et al., 2012; Kalkanci et al., 2012). Coutinho \& Paula (2000) avaliaram 30 isolados fúngicos provenientes de diferentes sítios anatômicos de cães com afecções e constataram que todas foram produtoras de proteases com Pz de 0,30. Outros estudos foram realizados por Coutinho (2005) e por Bona et al. (2012) por cepas oriundas de cães sem e com afecções, verificando que nestes últimos, a enzima foi produzida em $31 \%$ das amostras.

Os fatores de virulência não atuam separadamente. Acerca da função das proteases, alguns autores (Lima et al., 2004; Coutinho, 2005), não observaram diferença significativa entre os grupos sintomáticos e assintomáticos e concluíram exatamente que outros fatores contribuem para a agressão do hospedeiro. Outros autores como Bona et al. (2012) e Kalkanci et al. (2012) detectaram esta enzima e e comprovaram a alta patogenicidade dessas espécies.

A fosfolipase é considerada como fator importante para o processo de infecção, variando conforme a amostra e há a dependencia de outros fatores de virulência e do próprio hospedeiro. Ortiz et al. (2013) avaliaram a atividade da fosfolipase e protease de leveduras isoladas de cães sadios e com afecções e verificaram que das 95 cepas avaliadas para produção de protease 93 (97,89\%) apresentaram atividade em pH 6,8 e 77 (81,05\%) em pH 6,3, com diferentes valores de Pz. Cafarchia \& Otranto (2004) avaliaram a atividade da fosfolipase em diferentes sítios anatômicos de cães, e verificaram que cepas oriundas de animais com afecções apresentaram uma maior atividade fosfolipásica (93,9\%) quando comparado com cepas de cães saudáveis (10,6\%). Em estudo realizado por Machado et al. (2010) foi verificado que o número de cepas de M. pachydermatis que produziram fosfolipase foi estatisticamente maior para cães doentes do que para as estirpes encontradas em animais saudáveis.

Neste trabalho, dos 60 isolados testados para produção de fosfolipase 28 (46,66\%) foram negativas, 22 (36,66\%) forte positivas e 10 (16,66\%) positivas, ou seja, 32 (53,33\%) amostras são produtoras de fosfolipase, com índices de Pz variando de 0,22 a 0,937.

Brilhante et al. (2014) isolaram 28 cepas de Candida parapsilosis de animais clinicamente saudáveis, e verificaram que nenhuma das cepas foi produtora de fosfolipase. No presente estudo as únicas duas cepas desta espécie mostraram-se negativas para a produção desta enzima.

O gênero Rhodotorula sempre foi considerado não patogênico, mas tem sido apontado como levedura emergente nas infecções clínicas era (Goyal et al., 2008; Cunha et al., 2009) o que mostra a importância de isolamento, neste estudo. Além do isolamento da mucosa cérvico-vaginal, as cepas isoladas destacaram-se como fortes produtoras de fosfolipase.

A produção de protease e de fosfolipase determinadas para estes isolados é apenas um passo na determinação da virulência das mesmas. É necessário lembrar que a virulência depende de 
muitos fatores. Logo, os atributos que contribuem para virulência destas leveduras incluem adesão, formação de hifas, dimorfismo entre outras, incluindo a produção destas enzimas hidrolíticas extracelulares.

Das 60 cepas avaliadas 20 (33,33\%) foram produtoras de ambas enzimas, 14 (23,33\%) produtoras apenas para protease, 17 (28,33\%) apenas para fosfolipase e 9 (15,0\%) negativa para as duas.

Candida albicans é citada por diversos autores como sendo das maiores espécies produtoras de fosfolipase e protease (D’Éça Junior et al., 2011; Branco et al., 2012; Sachin et al., 2012). Esta nossa pesquisa corrobora com estes dados. O presente trabalho comprova o fato que, não apenas Candida albicans como também, Candida não albicans, aparecem com algum grau de importância neste cenário. Para produção de fosfolipase, foram positivas 57,14\% para C. tropicalis, 50\% para Candida albicans, e todas as cepas de C. famata, C. guilliermondii, C. glabrata, C. parapsilosis foram negativas. Para produção de protease, todas foram produtoras em C. albicans, C. parapsilosis, C. guilliermondii, 85,71\% em C. tropicalis, 33,33\% em C. glabrata e nenhuma em C. famata.

O conhecimento de micro-organismos potencialmente causadores de patologias presentes no trato reprodutivo de cadelas representa talvez a solução de inúmeros distúrbios ainda pouco explicados e controlados na medicina veterinária.

Em relação a produção de enzimas, as leveduras isoladas da mucosa cérvico-vaginal de cadelas hígidas produzem fosfolipase e/ou protease, apresentando algum grau de virulência. Além disso, deve-se considerar que inúmeras amostras isoladas neste trabalho apresentaram Pz compativel com a classificação fortemente postiva, o que sugere que essas amostras possuem potencial patogênico aos animais. Entretanto para o agente ser agressivo ao hospedeiro, também deve-se levar em consideração o estado imunológico, pois a maioria delas são leveduras oportunistas.

O isolamento das cepas agentes de possíveis distúrbios reprodutivos, a determinação das espécies e a avaliação dos fatores de virulência são imprescindíveis para uma boa compreensão dos casos clínicos, principalmente em casos recorrentes. Isso aponta a importância dos estudos destas leveduras já que assumem grande potencial patogênico para estes animais.

\section{Conclusões}

Candida spp, Malassezia pachydermatis, Rhodotorula spp e Trichosporon spp são leveduras normalmente presentes na micobiota cérvico-vaginal de cadelas hígidas. Não somente a espécie Candida albicans, mas outras espécies podem estar presentes no trato reprodutivo de cadelas. A espécie Malassezia pachydermatis, normalmente referida como presente na pelee no conduto auditivo de cães, faz parte da micobiota do trato reprodutivo destes animais.

Muitos fungos leveduriformes presentes no trato reprodutivo de cadelas são produtores de enzimas relacionadas com virulência, podendo ser fortes produtoras destas enzimas.

\section{Referências}

Bona, E., Telesca, S. U. P., \& Fuentefria, A. M. (2012). Ocurrence and identification of yeasts ind dogs external ear canal with and without oitis. Revista de Medicina Veterinária e Zootecia Córdoba, 17(2), 3059-3064.

Branco, P. V. G. C., Anjos, D. C. V., Nascimento, F. B., Vale, I. N. F., Azevedo, C. M. P. S., Monteiro, S. G., Figueiredo, P. D. M. S., \& Monteiro, C. D. A. (2012). Prevalência e produção de exoenzimas por espécies de Candida provenientes da mucosa bucal de pacientes com AIDS e indivíduos hígidos. Revista de Patologia Tropical, 41(4), 427-441. http://dx.doi.org/10.5216/rpt.v41i4.21703.

Brilhante, R. S. N., Rodrigues, T. J. S., Castelo-Branco, D. S. C. M., Teixeira, C. E. C., Macedo, R. B., Bandeira, S. P., Pereira de Alencar, L., Monteiro, A. J., Cordeiro, R. A., Bandeira, T. J. P. G., Moreira, J. L. B., Sidrim, J. J. C., \& Rocha, M. F. G. (2014). Antifungal susceptibility and virulence attibutes of animal-derived isolates of Candida parapasilosis complex. Journal of Medical Microbiology, 63(Pt 11), 1568-1572. http://dx.doi.org/10.1099/ imm.0.076216-0. PMid:25190736.

Cafarchia, C., \& Otranto, D. (2004). Association between phospholipase production by Malassezia pachydermatis and skin lesions. Journal of Clinical Microbiology, 42(10), 4868-4869. http://dx.doi.org/10.1128/JCM.42.10.48684869.2004. PMid:15472366.

Cleff, M. B., Xavier, M. O., Martins, A. A., Santin, R., \& Meireles, M. C. A. (2007). Caracterización de La microbiota leveduriforme residente em La vagina de perras em diferentes fases del ciclo estral. Archivos de Medicina Veterinaria, 39(2), 153-158. http://dx.doi.org/10.4067/50301-732X2007000200009. 
Corrêa, P. R., David, P. R. S., Peres, N. P., Cunha, K. C., \& Almeida, M. T. G. (2009). Caracterização fenotípica de leveduras isoladas da mucosa vaginal em mulheres adultas. Revista Brasileira de Ginecologia e Obstetrícia, 31(4), 177-181. http://dx.doi.org/10.1590/S0100-72032009000400004. PMid:19578672.

Coutinho, S. D. A. (2005). Malassezia pachydermatis: enzymes production in isolates from external ear canal of dogs with and without otitis. Arquivo Brasileiro de Medicina Veterinária e Zootecnia, 57(suppl 2), 149-153. http://dx.doi.org/10.1590/S0102-09352005000800003.

Coutinho, S. D., \& Paula, C. R. (2000). Proteinase, phospholipase, hyaluronidase and chondroitin-sulfatase production by Malassezia pachydermatis. Medical Mycology, 38(1), 73-76. http://dx.doi.org/10.1080/mmy.38.1.73.76. PMid:10746230.

Cunha, M. M. L., Santos, L. P. B., Dornelas-Ribeiro, M., Vermelho, A. B., \& Rozental, S. (2009). Identification, antifungal susceptibility and scanning electron microscopy of a keratinolytic strain of Rhodotorula mucilaginosa: a primary causative agent of onychomycosis. FEMS immunology and medical microbiology, 55(3), 396-403. PMid:19170752.

D’Éça Junior, A. D., Silva, A. F., Rosa, F. C., Monteiro, S. G., Figueiredo, P. M. S., \& Monteiro, C. A. (2011). Atividade diferencial in vitro de fosfolipases e proteinases ácidas de isolados clínicos de Candida. Revista da Sociedade Brasileira de Medicina Tropical, 44(30), 334-338. http://dx.doi.org/10.1590/S0037-86822011005000036. PMid:21901875.

Fraga, M. E., Baroni, F. A., Carvalho, M. G., Santos, A. G., \& Andrade, J. B. (2008). Microbiota de fluido cervicovaginal de cabras, cadelas e gatas. Revista Brasileira de Medicina Veterinária, 30(1), 50-54.

Goyal, R., Das, S., Arora, A., \& Aggarwal, A. (2008). Rhodotorula mucilaginosa as a cause of persistent femoral nonunion. Journal of Postgraduate Medicine, 54(1), 25-27. http://dx.doi.org/10.4103/0022-3859.39186. PMid:18296801.

Kalkanci, A., GüZel, A. B., Khalil, I. I. J., Aydin, M., Ilkit, M., \& Kuştimur, S. (2012). Yeast vaginistis during pregnancy:susceptibility testing of 13 antifungal drugs and boric acid and the detection of four virulence factors. Medical Mycology, 50(6), 585-593. http://dx.doi.org/10.3109/13693786.2012.662597. PMid:22369624.

Kumar, G., Kumar, S. J., \& Menon, T. (2006). Phospholipase and proteinase activities of clinical isolates of Candida from immunocompromised patients. Mycopathologia, 161(4), 213-218. http://dx.doi.org/10.1007/s11046-0050157-4. PMid:16552483.

Kurtzman, C., \& Fell, J. W. (1998). The Yeasts. A taxonomic study. 4th ed. Amsterdam: Elsevier.

Kurtzman,C., Fell, J.W. \& Boekhout,T. (2011). The yeasts, a taxonomic study (5th ed., 2354 p.) New York: Elsevier.

Lima, T. D., Fernandes, O. F. L., Souza, L. K. H., Passos, X. S., \& Silva M R R. (2004). Candida albicans de mucosa vaginal: morfotipagem e produção de proteinase. Revista de Patologia Tropical, 33(1), 65-70.

Machado, M. L., Cafarchia, C., Otranto, D., Ferreira, R. R., Bianchi, S. P., Latrofa, M. S., Parisi, A., \& Ferreiro, L. (2010). Genetic variability and phospholipase production of Malassezia pachydermatis isolated from dogs with diverse grades of skin lesions. Medical Mycology, 48(6), 889-892. http://dx.doi.org/10.3109/13693780903532080. PMid:20105099.

Markey, B., Leonard, F., Archambault, M., Cullinane, A., \& Maguire, D. (2013). Clinical Veterinary Microbiology (2nd ed.). USA: Mosby Elsevier.

Ortiz, G., Martín, M. C., Carrillo-Muñoz, A. J., \& Payá, M. J. (2013). Producción de fosfolipasa y proteinasa em cepas de Malassezia pachydermatis aisladas de perros com otitis y sin otitis. Revista Iberoamericana de Micologia, 30(4), 235-238. http://dx.doi.org/10.1016/j.riam.2013.01.006. PMid:23428748.

Price, M. F., Wilkinson, I. D., \& Gentry, L. O. (1982). Plate method for detection os phospholipase activity in Candida albicans. Sabouraudia, 20(1), 7-14. http://dx.doi.org/10.1080/00362178285380031. PMid:7038928.

Rippon, J. N. (1988). The pathogenic fungi and the pathogenic actinomycetes. In Rippon, J. W.. Medical mycology: the pathogenic fungi and the pathogenic actinomycetes. 797 p.). Philadelphia: W.B. Saunders Co.

Sachin, C. D., Ruchi, K., \& Santosh, S. (2012). In vitro evaluation of proteinase, phospholipase and haemolysin activities of Candida species isolated from clinical specimens. International Journal of Medicine and Biomedical Research, 1(2), 153-157. http://dx.doi.org/10.14194/ijmbr.1211.

Stein, M., Castro, L. L D., Guiot, E. G., Silva, L. G. C., Silveira, G. R., Cleff, M. B., \& Schramm, R. C. (2009). Avaliação da microbiota vaginal de fêmeas caninas relacionadas com o ciclo estral. Brazilian Journal of Microbiology, 36, 201-204.

Tedesco, J. J. A., Britto, I. S. W., \& Rodrigues, L. P. (2006). Vulvovaginites na gestação. Revista da Associação de Obstetrícia e Ginecologia do Estado de São Paulo, 63(2), 1-2.

Tizard, I. R. (1998). Hipersensibilidade do tipo IV: Rejeição de transplantes. In I. R. Tizard. Imunologia veterinária: uma introdução (pp. 408-419). São Paulo, Roca.

Verma, S., Katoch, R. C., Jand, S. K., Sharma, B. M., \& Nigam, P. (1999). Myconitoc flora of female genitalia of buffaloes and cows with reproductive disorders. Veterinary Research Communications, 23(6), 337-341. http:// dx.doi.org/10.1023/A:1006381523941. PMid:10543363. 